\title{
Archéopages
}

Archéopages

Archéologie et société

34 | 07/2012

Campagnes

\section{Modélisation de terrain par prise de vue embarquée}

Régis Touquet

\section{OpenEdition}

1 Journals

Édition électronique

URL : https://journals.openedition.org/archeopages/423

DOI : 10.4000/archeopages.423

ISSN : 2269-9872

Éditeur

INRAP - Institut national de recherches archéologiques préventives

Édition imprimée

Date de publication : 1 février 2012

Pagination : 98-99

ISSN : 1622-8545

\section{Référence électronique}

Régis Touquet, " Modélisation de terrain par prise de vue embarquée », Archéopages [En ligne], 34 |

07/2012, mis en ligne le 01 juillet 2012, consulté le 21 janvier 2022. URL : http://

journals.openedition.org/archeopages/423 ; DOI : https://doi.org/10.4000/archeopages.423

(C) Inrap 
confirmer ou infirmer des hypothèses de contenants et réaliser des illustrations [ill. 2]. De plus, tous les modèles peuvent être fournis aux différents acteurs de l'étude au format pdf $3 \mathrm{D}$, facilement lisible avec Adobe Reader ou Acrobat [ill. 3], qui permet de visionner les acquisitions $3 \mathrm{D}$ à l'échelle, de faire des mesures et de pratiquer des coupes. La photogrammétrie a ici permis de trouver une nouvelle approche d'étude des incinérations. Différentes hypothèses de formes de contenant peuvent être confrontées à une représentation virtuelle, afin de les valider - ou non - et de les illustrer. De plus, la forme et les couleurs de faits archéologiques, qui seront à terme démantelés par la fouille, peuvent être sauvegardées. Enfin, cet enregistrement peut alimenter une base de données utile pour des études ultérieures.

\section{Modélisation de terrain par prise de vue embarquée}

Régis Touquet, Inrap

Une campagne de prise de vues aériennes par drone a été réalisée sur l'opération archéologique de Morigny-Champigny (Essonne), couvrant $5000 \mathrm{~m}^{2}$ et divisée en trois zones distinctes. La couverture photographique complète de l'emprise et l'importante quantité d'images produites par un tel procédé a permis d'exploiter les clichés dans un travail de modélisation de leur géométrie. Il est en effet possible, grâce à la technique de la photogrammétrie, d'exploiter des images diverses tant pour leur échelle que par les types d'appareils de prises de vues. Outre la possibilité de mettre à l'épreuve cette technique sur une grande surface, le but était de créer un modèle numérique de terrain précis des différentes zones de fouilles et ce, uniquement à partir des documents photographiques. La société Kyuaero a réalisé les prises de vues aériennes à l'aide d'un drone commandé depuis le sol. Il s'agissait d'obtenir des photographies zénithales ainsi que des photographies angulaires, le drone survolant alors de manière concentrique les emprises à traiter. Les clichés ont été pris en mode rafale, à environ 20 mètres du sol, à raison d'une photographie par seconde. Le couvert nuageux entrainant une faible lumière le jour des prises de vue, la multiplication des images a ainsi été privilégiée. Un fond photographique d'une centaine de clichés exploitables par zones a été obtenu. Ces données raster brutes, d'une définition de 12 millions de pixels, ont ensuite été soumises à des solutions logicielles. Chaque cliché réalisé sur les zones de l'emprise a été traité de manière indépendante par les logiciels libres Bundler, PMVS, CMVS et Meshlab, afin d'en acquérir un modèle $3 \mathrm{D}$ à haute densité de points. Trois Modèles Numériques de Terrain (MNT) ont par la suite été calculés, couvrant des surfaces au sol de 600,1900 et $2500 \mathrm{~m}^{2}$. Un MNT se représente par des faces triangulaires modélisées en $3 \mathrm{D}$, qui traduisent le relief calculé. Le nombre de ces faces dépend davantage de la pertinence géométrale des sources de données utilisées que de la surface du modèle. Les logiciels d'acquisition $3 \mathrm{D}$ précités peuvent générer, selon la pertinence des informations, plusieurs millions de faces, offrant ainsi une visualisation très précise et fidèle des sujets traités. Le modèle obtenu doit être orienté selon le système de projection de l'opération. En amont du traitement, des points remarquables sont ainsi levés sur le terrain et déterminés par coordonnées. Ces données correspondent à des points d'angles ou d'arêtes identifiables sur le MNT calculé. Le géo-référencement du modèle en projection et en élévation permet ainsi d'analyser, de manière précise, la topographie existante et de la soumettre aux différents outils de visualisation. Des cartes en relief [ill. 1] des trois zones ont été dressées, facilitant ainsi la lecture topographique et la comparaison des altitudes d'une zone à l'autre. De plus, durant la phase de fouille et de post-fouille, un nombre infini de profils peuvent être exportés du modèle [ill. 2], traduisant graphiquement en $2 \mathrm{D}$ les informations du relief. Ces profils de terrains permettent également un contrôle de précision du MNT en le comparant à un levé traditionnel effectué par le topographe [ill. 3]. Toutefois, la résolution obtenue par la photogrammétrie s'avère beaucoup plus pertinente, car elle génère un nombre de points topographiques important, trop lourd à mettre en œuvre avec un levé au tachéomètre.

La confrontation des différentes couches superposables que sont le MNT, le plan-masse du site et les images géo-référencées issues de la photogrammétrie facilite la lecture des zones modélisées et permet une approche tridimensionnelle des problématiques. L'analyse des pentes, l'étude du microrelief, le calcul des cubatures font partie des outils utiles au responsable d'opération.

La procédure d'acquisition étant simple à mettre en œuvre, elle peut être répétée et permettre ainsi la comparaison précise de différents modèles successifs. Les niveaux d'occupation ou de décapage peuvent alors être documentés au fil de l'opération archéologique. La caractérisation des aspects du relief peut devenir systématique et intégrer une réflexion plus large sur l'implantation des occupations humaines.

\section{Relevé en 3D d'une structure souterraine} médiévale

Chhavy-Cyril Tan, Service archéologique départemental d'Indre-et-Loire Laurent Fournier, Inrap

La découverte, au cours de l'été 2009, d'une structure souterraine, annexe d'un habitat médiéval, sur le site d'Ormes « Le Bois d'Ormes » (Loiret) (Fournier, 2011) a conduit à la mise en 